Tropical Journal of Pharmaceutical Research January 2017; 16 (1): 203-210

ISSN: $1596-5996$ (print); 1596-9827 (electronic)

(C) Pharmacotherapy Group, Faculty of Pharmacy, University of Benin, Benin City, 300001 Nigeria.

All rights reserved.

Available online at http://www.tjpr.org

Original Research Article

http://dx.doi.org/10.4314/tjpr.v16i1.27

\title{
Synthesis and antioxidant potential of some biscoumarin derivatives
}

\author{
Anum Sahar ${ }^{1,2}$, Zulfiqar Ali Khan ${ }^{1 *}$, Matloob Ahmad ${ }^{1}$, Ameer Fawad Zahoor ${ }^{1}$, \\ Asim Mansha ${ }^{1}$ and Ahsan Iqbal ${ }^{1}$ \\ ${ }^{1}$ Department of Chemistry, Government College University, Allama lqbal Road-38000, ${ }^{2}$ Department of Chemistry and \\ Biochemistry, University of Agriculture, Faisalabad, Pakistan
}

*For correspondence: Email: zulfiqar_chem@yahoo.co.in

Revised accepted: 13 December 2016

\begin{abstract}
Purpose: To synthesize and screen some 3-substituted-bis-2H-chromen-2t-ones in a search for possible new antioxidants for use in food and pharmaceuticals industries.

Methods: 2-Hydroxycoumarin was allowed to react with various substituted aromatic aldehydes in the presence of base as a catalyst to obtain to obtain 3-substituted-bis-2H-chromen-2t-ones. The chemical structures of the synthesized compounds were confirmed by Fourier transform infra-red (FT-IR) spectroscopy, proton nuclear magnetic resonance $\left({ }^{1} H\right.$ NMR), mass spectrometry (MS) and elemental analysis. The synthesized biscoumarin derivatives were screened by three parallel antioxidant assays, namely, 2,2-diphenyl-1-picrylhydrazyl (DPPH) free radical scavenging activity, reducing power, and linoleic acid lipid peroxidation. Further, the half minimal inhibitory concentration $\left(I C_{50}\right)$ of the compounds was also determined. Butylatedhydroxy toluene (BHT) was used as a standard reference in the antioxidant assay.

Results: A library of ten biscoumarin analogues (3a-3j) were synthesized and evaluated for their antioxidant potential. The highest DPPH activity was shown by 3,3'-(3-hydroxy-4methoxyphenylmethylene)-bis-4-hydroxy-2H-chromen-2-one (3g) and 3,3'-(4-methoxyphenylmethylene)-bis-4-hydroxy-2H-chromen-2-one (3h) with I $\mathrm{C}_{50}$ values of $53.33 \pm 6.12$ and $68.44 \pm 2.67$ $\mu \mathrm{g} / \mathrm{mL}$, respectively, compared to the standard compound BHT (butylatedhydroxy toluene) with $I_{50}$ of $71.00 \pm 0.82 \mu \mathrm{g} / \mathrm{mL}$. The strongest inhibition of linoleic acid peroxidation was by 3,3'-(3-hydroxy-4methoxyphenylmethylene)-bis-4-hydroxy-2H-chromen-2-one (3g) and 3'-(4-hydroxy-3-methoxyphenylmethylene)-bis-4-hydroxy-2H-chromen-2-one (3d) with inhibition values of 70.34 and $69.87 \%$, respectively. Compound $3 g$ demonstrated the highest reducing power with an absorbance of 0.49 at 10 $\mathrm{mg} / \mathrm{mL}$ concentration $(p<0.001)$. It was observed that the change in substitution greatly affected the antioxidant potential of the compounds, with the presence of the methoxy group at position-4 of aryl moiety along with 2-hydroxycoumarin being critical for antioxidant activity.

Conclusion: The synthesized biscoumarin derivatives, $\mathbf{3 g}$ and $\mathbf{3 h}$, are superior antioxidants to standard $(B H T)$ and are suitable lead molecules for further development.
\end{abstract}

Keywords: Antioxidant, Biscoumarins, DPPH, Ferric reducing assay, linoleic acid lipid peroxidation assay

Tropical Journal of Pharmaceutical Research is indexed by Science Citation Index (SciSearch), Scopus, International Pharmaceutical Abstract, Chemical Abstracts, Embase, Index Copernicus, EBSCO, African Index Medicus, JournalSeek, Journal Citation Reports/Science Edition, Directory of Open Access Journals (DOAJ), African Journal Online, Bioline International, Open-J-Gate and Pharmacy Abstracts

\section{INTRODUCTION}

Free radicals are part of groups of molecules collectively called reactive oxygen species (ROS) which includes superoxide anion $\left(\mathrm{O}_{2}{ }^{\circ}\right)$, hydroxyl radical $\left({ }^{\circ} \mathrm{OH}\right)$, perhydroxyl radical $\left(\mathrm{HO}_{2}{ }^{\circ}\right)$, nitric oxide and other species such as singlet oxygen $\left({ }^{1} \mathrm{O}_{2}\right)$, hydrogen peroxide $\left(\mathrm{H}_{2} \mathrm{O}_{2}\right)$, hypochlorous 
acid $(\mathrm{HOCl})$ and peroxynitrite $(\mathrm{ONOO})$ etc [1]. ROS are produced as a result of cellular metabolism and physiological activities and play imperative roles in cell signaling, gene expression, apoptosis and ion transportation. However, excessive amounts of ROS (a condition called oxidative stress) can have deleterious effects on health by damaging molecules such as protein, lipid, RNA and DNA [2]. ROS interact with cells of living bodies and damage them to cause early aging of skin and other serious chronic diseases such as cancer, brain dysfunction and immune system decline [3]. A balance between oxidant and antioxidant in intracellular systems is vital for normal cell function [4]. Therefore, antioxidants are added in food to protect it from oxidative deterioration and helps in the treatment of many diseases such as anti-aging medicine, cancer, antitumor and antiHIV therapy, and as stimulants for central nervous system, antibacterial and antiinflammatory agents $[3,5]$.

Plants are the major source of antioxidant compounds and they help to prevent damage caused by the reactive oxygen species [6]. Antioxidants from natural sources are not enough to fulfill the increasing requirements of growing world population therefore it is highly desirable to synthesize and search for new and effective synthetic antioxidants. The literature reveals that coumarin-based compounds exhibit strong antioxidant activities and they occur in vegetation [7], with several pharmacological applications such as enzyme inhibitors [8,9], antioxidant activity [10], anticancer [11], anti-HIV [12], cytotoxicity [13] and anti-inflammatory activity [14]. Structure activity relationship studies reveal that the biological activities of these compounds are greatly affected by the change in structure and various research groups have reported the synthesis of biologically active molecules bearing coumarin moiety $[15,16]$. In this work, ten biscoumarin derivatives were synthesized and screened for antioxidant potential by three parallel assays namely DPPH (1,1-diphenyl-2picrylhydrazyl) radical scavenging activity, reducing power and linoleic acid lipid<smiles>[R2]c1ccc(C=O)cc1[R2]</smiles>

peroxidation. The synthesized compounds showed moderate to excellent antioxidant potential depending on the electronic nature of substituents attached to the aryl moiety.

\section{EXPERIMENTAL}

All chemicals, reagents and solvents used in this study were purchased from Sigma-Aldrich and Alfa Aesar. The proton nuclear magnetic resonance ( ${ }^{1} \mathrm{H}$ NMR) was recorded in $\mathrm{CDCl}_{3}$ using Bruker AC400 (400 MHz). The chemical shifts are reported in ppm and coupling constants have been measured in Hertz $(\mathrm{Hz})$. Melting points were measured on Buchi 434 melting point apparatus and were uncorrected. Reactions were monitored on $2 \times 5 \mathrm{~cm}$ aluminum sheets silica gel $60 \mathrm{~F} 254$ to a thickness $0.25 \mathrm{~mm}$ (Merck). The chromatograms were visualized under the ultra-violet light (254-366 nm) or iodine vapours. IR spectra ( $\mathrm{KBr}$ discs or $\mathrm{MeOH})$ were measured on a Bruker FT-IR IFS48 spectrophotometer. The UV-Visible spectrophotometer model 5000, Irmico, UK was used for absorbance measurements in the antioxidant assays. Low resolution mass spectra (EI-MS) were recorded on Finnigan MAT-311A Germany.

Biscoumrins derivatives (3a-j) were synthesized according to reported procedure [17] by reaction of 4-hydroxycoumarins (1) with aromatic aldehydes $(\mathbf{2 a}-\mathbf{j})$. The reaction was carried out in the presence of catalytic amount of base (few drops) and absolute ethanol as a solvent at room temperature (Figure 1). The synthesized compounds were obtained in good to excellent yields (35 - 97\%).

\section{General procedure for the synthesis of compounds (3a-j)}

The 4-hydroxycoumarin 1 (2 $\mathrm{mM}$ ) was condensed with different aromatic aldehydes (2aj) $(1 \mathrm{mM})$ in absolute ethanol $(10 \mathrm{~mL})$ as a solvent.

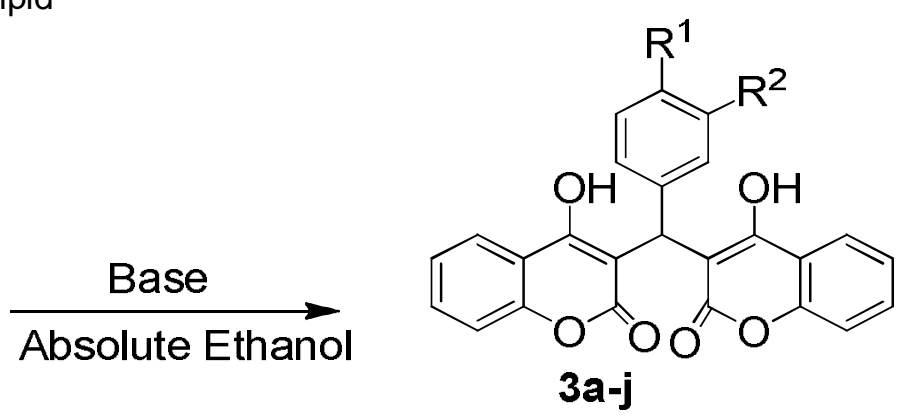

Figure 1: Synthesis of biscoumarin derivatives $3 a-j$ 
Table 1: Reaction time and yield of biscoumarins (3a-j)

\begin{tabular}{lllcc}
\hline Compound no. & $\mathbf{R}^{1}$ & $\mathbf{R}^{2}$ & Reaction time (h) & Yield (\%) \\
\hline $\mathbf{3 a}$ & $-\mathrm{H}$ & $-\mathrm{H}$ & 24 & 62 \\
$\mathbf{3 b}$ & $-\mathrm{Cl}$ & $-\mathrm{H}$ & 24 & 66 \\
$\mathbf{3 c}$ & $-\mathrm{H}$ & $-\mathrm{Cl}$ & 26 & 95 \\
$\mathbf{3 d}$ & $-\mathrm{OH}$ & $-\mathrm{OCH}_{3}$ & 48 & 46 \\
$\mathbf{3 e}$ & $-\mathrm{N}\left(\mathrm{CH}_{3}\right)_{2}$ & $-\mathrm{H}$ & 30 & 35 \\
$\mathbf{3 f}$ & $-\mathrm{H}^{\mathbf{H}}$ & $-\mathrm{OH}$ & 48 & 92 \\
$\mathbf{3 g}$ & $\mathrm{OCH}_{3}$ & $-\mathrm{OH}$ & 48 & 38 \\
$\mathbf{3 h}$ & $\mathrm{OCH}_{3}$ & $-\mathrm{H}$ & 36 & 97 \\
$\mathbf{3 i}$ & $-\mathrm{OH}^{3 \mathbf{H}}$ & $-\mathrm{H}$ & 48 & 92 \\
$\mathbf{3 j}$ & $-\mathrm{OCH}_{3}$ & $-\mathrm{OCH}_{3}$ & 24 & 40 \\
\hline
\end{tabular}

The ten biscoumarin analogues (3a-j) were successfully prepared according to Scheme 1.

A small amount of base piperidine $/ N$ methylmorpholine (4-5drops) were added to this solution and stirred for $24-48 \mathrm{~h}$ at room temperature. The course of the reaction was monitored by thin layer chromatography. After the completion of reaction, distilled water was added and resulting solid was filtered and washed with water and ethanol to furnish the product. The crude product was purified by recrystallization in ethanol.

\section{DPPH radical scavenging activity}

Free radical scavenging activity (RSA) of biscoumarin derivatives was measured by a method reported by Hussain et al [3]. Different concentrations $(20,40,60,80,100 \mu \mathrm{g} / \mathrm{mL})$ of compound were prepared and $3 \mathrm{~mL}$ of each concentration solution was added to $1 \mathrm{ml}$ of 0.1 $\mathrm{mM}$ DPPH ethanolic solution and the solutions were maintained for $30 \mathrm{~min}$ in dark and absorbance was measured at $517 \mathrm{~nm}$. Samples without compound and 2,6-ditert-butyl-4methylphenol (BHT) were taken as negative and positive controls, respectively, and RSA calculated as inhibition as in Eq 1.

Inhibition $(\%)=\left(1-A_{1} / A_{0}\right) 100$

$A_{1}=$ absorbance of compounds, $A_{0}=$ absorbance of negative control

\section{Reducing power assay}

Reducing capacity of biscoumarin derivatives was assessed by using Halliwell \& John method [17]. To a $1 \mathrm{~mL}$ of different concentrations $(2,4$, $6,8,10 \mathrm{mg} / \mathrm{mL}$ ) of compound were added in phosphate buffer (2.5 mL, pH 6.6) and potassium ferricyanide $(2.5 \mathrm{~mL}, 1 \%)$. This solution was maintained at $50^{\circ} \mathrm{C}$ for $20 \mathrm{~min}$. After that, trichloroacetic acid $(2.5 \mathrm{~mL}, 10 \%)$ was added to this solution and centrifuged for $10 \mathrm{~min}$ at 680 rpm. A $2.5 \mathrm{~mL}$ aliquot of upper layer was collected and mixed with distilled water $(2.5 \mathrm{~mL})$ and ferric chloride $(0.5 \mathrm{~mL}, 0.1 \%)$ and absorbance was measured at $700 \mathrm{~nm}$. BHT was taken as positive control.

\section{Antioxidant activity in linoleic acid system}

The antioxidant capacity of biscoumarin derivatives in the linoleic acid system was assessed by a literature procedure [3]. To $0.2 \mathrm{~mL}$ of linoleic acid emulsion $(25 \mathrm{mg} / \mathrm{mL}$ in $99 \%$ ethanol) and $0.4 \mathrm{~mL}$ of phosphate buffer $(0.05 \mathrm{M}$, $\mathrm{pH}$ 7.4) were added $0.2 \mathrm{~mL}(100 \mu \mathrm{g} / \mathrm{mL})$ of the compound. The resultant mixture was incubated at $40^{\circ} \mathrm{C}$. After each $24 \mathrm{~h}, 0.1 \mathrm{~mL}$ sample was taken and mixed with ammonium thiocyanate (3 $\mathrm{mL}, 70 \%)$ and ferrous chloride $(0.1 \mathrm{~mL}, 0.02 \mathrm{M}$ in $3.5 \% \mathrm{HCl}$ ). Exactly after $3 \mathrm{~min}$, the absorbance of this solution was taken at $500 \mathrm{~nm}$ and $\mathrm{BHT}$ was used as positive control.

\section{Statistical analysis}

Data from groups were statistically analyzed by univariate ANOVA followed by Bonferroni multiple comparison (post-hoc) test using IBM SPSS software version 23. Differences were considered significantly significant at $p<0.05$. The data are presented as mean \pm standard error of the mean (SEM, $n=3$ ).

\section{RESULTS}

The structures of these compounds were confirmed by ${ }^{1} \mathrm{H}$ NMR, mass spectrometry, FT-IR and $\mathrm{CHN}$ analysis. The spectroscopic data of title compounds (3a-j) are presented as follows:

\section{3, 3'-Phenylmethylene-bis-4-hydroxy-2H- chromen-2-one (3a)}

Yield: $62 \% ; \mathrm{R}_{f}=0.65$ (ethyl acetate), UV (DMF) $\lambda_{\max }: 304 \mathrm{~nm}$; IR $\left(\mathrm{v}_{\max }, \mathrm{KBr}, \mathrm{cm}^{-1}\right),: 3450,2949$, 1673, 1538; ${ }^{1} \mathrm{HNMR}\left(400 \mathrm{MHz}, \mathrm{CDCl}_{3}\right)$ : $\delta 11.67$ $(2 \mathrm{H}, \mathrm{s}, \mathrm{OH}), 7.96-7.15\left(13 \mathrm{H}, \mathrm{m}, \mathrm{H}_{\mathrm{Ar}}\right), 6.01(1 \mathrm{H}$, $\mathrm{s}, \mathrm{CH}) ; \mathrm{MS} \mathrm{m} / \mathrm{z}: 413(2), 412\left(\mathrm{M}^{+}, 5\right), 161.02$ 
(20), 117 (100), 77 (34), 51 (8); Anal. data calc. for $\mathrm{C}_{25} \mathrm{H}_{16} \mathrm{O}_{6}$ : C, 72.81; $\mathrm{H}, 3.91 ; \mathrm{O}, 23.28$. found: C, 72.79; H, 3.93; O, 23.27.

\section{3, 3'-(4-Chlorophenylmethylene)-bis-4- hydroxy-2H-chromen-2-one (3b)}

Yield: $66 \% ; \mathrm{R}_{f}=0.47$ (ethyl acetate), UV (DMF) $\lambda_{\max }: 302 \mathrm{~nm}$; IR (KBr) $\mathrm{v}_{\max }, \mathrm{cm}^{-1}: 3350,2956$, 1617, 1564; ${ }^{1} \mathrm{HNMR}\left(400 \mathrm{MHz} \mathrm{CDCl}_{3}\right): \delta 11.64$ $(2 \mathrm{H}, \mathrm{s}, \mathrm{OH}), 7.94-7.24\left(12 \mathrm{H}, \mathrm{m}, \mathrm{H}_{\mathrm{Ar}}\right), 6.07(1 \mathrm{H}$, $\mathrm{s}, \mathrm{CH}) ; \mathrm{MS}(\mathrm{m} / \mathrm{z}): 448(30) ; 446\left(\mathrm{M}^{+}, 11\right), 111$ (100), 113 (33),117 (76). Anal. Data calc. for $\mathrm{C}_{25} \mathrm{H}_{15} \mathrm{ClO}_{6}$ : C, 67.20; H, 3.38; Cl, 7.93; O, 21.48 . found: C, 67.22; $\mathrm{H}, 3.36 ; \mathrm{Cl}, 7.94 ; \mathrm{O}, 21.47$.

\section{3,3'-(3-Chlorophenylmethylene)-bis-4- hydroxy-2H-chromen-2-one (3c)}

Yield: $95 \% ; R_{f}=0.54$ (ethyl acetate), UV (DMF) $\lambda_{\max }: 302 \mathrm{~nm}$; IR $(\mathrm{KBr}) \mathrm{v}_{\max }, \mathrm{cm}^{-1}: 3450,2951$, 1601, 1520; ' $\mathrm{HNMR}\left(400 \mathrm{MHz}, \mathrm{CDCl}_{3}\right.$ ): $\delta 11.59$ $(2 \mathrm{H}, \mathrm{s}, \mathrm{OH}), 7.97-7.23\left(12 \mathrm{H}, \mathrm{m}, \mathrm{H}_{\mathrm{Ar}}\right), 6.10(1 \mathrm{H}$, $\mathrm{s}, \mathrm{CH}) ; \mathrm{MS}(\mathrm{m} / \mathrm{z}): 448(25, \mathrm{M}+2), 446\left(\mathrm{M}^{+}, 7\right)$, 410 (14), 325 (8), 249 (100), 162 (19); Anal. Data calc. for $\mathrm{C}_{25} \mathrm{H}_{15} \mathrm{ClO}_{6}$ : C, 67.20; $\mathrm{H}, 3.38 ; \mathrm{Cl}, 7.93$; O, 21.48. found: C, 67.22; $\mathrm{H}, 3.35 ; \mathrm{Cl}, 7.96 ; \mathrm{O}$, 21.48 .

\section{3, 3'-(4-Hydroxy-3-methoxyphenylmethylene)- bis-4-hydroxy-2H-chromen-2-one $(3 \mathrm{~d})$}

Yield: $46 \% ; R_{f}=0.89$ (ethyl acetate), UV (DMF) $\lambda_{\max }: 304 \mathrm{~nm}$; IR $(\mathrm{KBr}) \mathrm{v}_{\max }, \mathrm{cm}^{-1}: 3529,2941$, 1661, 1566; 'HNMR (400 MHz, $\mathrm{CDCl}_{3}$ ): $\delta 11.48$ $(2 \mathrm{H}, \mathrm{s}, \mathrm{OH}), 11.16(1 \mathrm{H}, \mathrm{s}, \mathrm{OH}), 7.99-7.26(11 \mathrm{H}$, $\left.\mathrm{m}, \mathrm{H}_{\mathrm{Ar}}\right), 6.09(1 \mathrm{H}, \mathrm{s}, \mathrm{CH}), 3.73\left(3 \mathrm{H}, \mathrm{s}, \mathrm{OCH}_{3}\right) ; \mathrm{MS}$ (m/z): 459 (1), 458.1 (10), 314 (10), 161 (34), 136 (100), 121 (25), 76 (10); Anal. Data calc. for $\mathrm{C}_{26} \mathrm{H}_{18} \mathrm{O}_{8}$ : C, 68.12; $\mathrm{H}, 3.96$; O, 27.92. found: $\mathrm{C}$, $68.14 ; \mathrm{H}, 3.94 ; \mathrm{O}, 27.91$.

\section{3, 3'-((4-(Dimethylamino)phenyl)methylene)- bis-4-hydroxy-2H-chromen-2-one (3e)}

Yield: $35 \% ; \mathrm{R}_{f}=0.92$ (ethyl acetate), UV (DMF) $\lambda_{\max }: 302 \mathrm{~nm}$; IR $(\mathrm{KBr}) \mathrm{v}_{\max }, \mathrm{cm}^{-1}: 3449,2903$, 1620, 1534, 1315; ${ }^{1} \mathrm{HNMR}\left(400 \mathrm{MHz} \mathrm{CDCl}_{3}\right): \delta$ $11.45(2 \mathrm{H}, \mathrm{s}, \mathrm{OH}), 7.99-7.24\left(12 \mathrm{H}, \mathrm{m}, \mathrm{H}_{\mathrm{Ar}}\right), 6.17$ $(1 \mathrm{H}, \mathrm{s}, \mathrm{CH}), 3.78\left(6 \mathrm{H}, \mathrm{s}, \mathrm{CH}_{3}\right) ; \mathrm{MS}(\mathrm{m} / \mathrm{z}): 456$ (2) 455 (13), 161 (26), 121 (100), 76 (2); Anal. Data calc. for $\mathrm{C}_{27} \mathrm{H}_{21} \mathrm{NO}_{6}$ : C, 71.20; $\mathrm{H}, 4.65 ; \mathrm{N}, 3.08$; O, 21.08. found: C, 71.22; H, 4.663; N, 3.07; O, 21.09 .

\section{3,3'-(3-Hydroxyphenylmethylene)-bis-4- hydroxy-2H-chromen-2-one (3f)}

Yield: $92 \% ; R_{f}=0.74$ (ethyl acetate), UV (DMF) $\lambda_{\max }: 302 \mathrm{~nm}$; IR $(\mathrm{KBr}) \mathrm{v}_{\max }, \mathrm{cm}^{-1}: 3340,2949$,
1643, 1532; ${ }^{1} \mathrm{HNMR}\left(400 \mathrm{MHz}, \mathrm{CDCl}_{3}\right): \delta 11.57$ $(2 \mathrm{H}, \mathrm{s}, \mathrm{OH}), 11.21(1 \mathrm{H}, \mathrm{s}, \mathrm{OH}), 7.95-6.85(12 \mathrm{H}$, $\left.\mathrm{m}, \mathrm{H}_{\mathrm{Ar}}\right), 6.03(1 \mathrm{H}, \mathrm{s}, \mathrm{CH})$; Ms (m/z): $429(1), 428$ $\left(\mathrm{M}^{+}, 11\right), 307(3), 265$ (23), 221 (2), 162 (56), 120.1 (100), 76 (2); Anal. Data calc. for $\mathrm{C}_{25} \mathrm{H}_{16} \mathrm{O}_{7}$ : C, 70.09; $\mathrm{H}, 3.76$; O, 26.14; found: C, 70.08; H, 3.77; O, 26.15.

3, 3'-(3-Hydroxy-4-methoxyphenylmethylene)bis-4-hydroxy-2H-chromen-2-one $(3 \mathrm{~g})$

Yield: $38 \% ; R_{f}=0.69$ (ethyl acetate), UV (DMF) $\lambda_{\text {max }}: 304 \mathrm{~nm}$; IR $(\mathrm{KBr}) \mathrm{v}_{\max }, \mathrm{cm}^{-1}: 3529,2941$, 1661, 1566; ' ${ }^{\mathrm{HNMR}}$ (400 MHz, $\mathrm{CDCl}_{3}$ ): $\delta 11.56$ $(2 \mathrm{H}, \mathrm{s}, \mathrm{OH}), 11.22(1 \mathrm{H}, \mathrm{s}, \mathrm{OH}), 7.99-6.75(11 \mathrm{H}$, $\left.\mathrm{m}, \mathrm{H}_{\mathrm{Ar}}\right), 6.12(1 \mathrm{H}, \mathrm{s}, \mathrm{CH}), 3.11\left(3 \mathrm{H}, \mathrm{s}, \mathrm{OCH}_{3}\right)$; MS (m/z): 459 (21), 458 (2), 161 (34), 136.1 (10), 117 (100), 76 (3); Anal. Data calc. for $\mathrm{C}_{26} \mathrm{H}_{18} \mathrm{O}_{8}$ : C, 68.12; H, 3.96; O, 27.92; found: C, 68.14; H, $3.94 ; 0,27.93$.

\section{3,3'-(4-Methoxyphenylmethylene)-bis-4- hydroxy-2H-chromen-2-one (3h)}

Yield: $97 \% ; R_{f}=0.79$ (ethyl acetate), UV (DMF) $\lambda_{\max }$ : $302 \mathrm{~nm}$; IR (KBr) $\mathrm{v}_{\max }, \mathrm{cm}^{-1}: 3304,2947$, 1678, 1562; ${ }^{1} \mathrm{HNMR}$ (400 MHz, $\mathrm{CDCl}_{3}$ ): $\delta 12.37$ $(2 \mathrm{H}, \mathrm{s}, \mathrm{OH}), 7.60-6.74\left(12 \mathrm{H}, \mathrm{m}, \mathrm{H}_{\mathrm{Ar}}\right), 6.04(1 \mathrm{H}$, $\mathrm{s}, \mathrm{CH}), 3.15\left(3 \mathrm{H}, \mathrm{s}, \mathrm{OCH}_{3}\right) ; \mathrm{MS}(\mathrm{m} / \mathrm{z}): 443(6)$, $442\left(\mathrm{M}^{+}, 76\right), 321$ (12), 281 (20), 265 (17), 249 (100), 162 (64), 120 (74); 76 (5); Anal. Data calc. for $\mathrm{C}_{26} \mathrm{H}_{18} \mathrm{O}_{7}$ : C, 70.58; $\mathrm{H}, 4.10 ; \mathrm{O}, 25.31$; found: C, 70.56; H, 4.11; O, 25.32.

\section{3,3'-(4-Hydroxyphenylmethylene)-bis-4- hydroxy-2H-chromen-2-one (3i)}

Yield: $92 \% ; R_{f}=0.67$ (ethyl acetate), UV (DMF) $\lambda_{\max }: 304 \mathrm{~nm}$; IR $(\mathrm{KBr}) \mathrm{v}_{\max }, \mathrm{cm}^{-1}: 3445,2929$, 1663, 1563; ${ }^{1} \mathrm{HNMR}$ (400 MHz, $\mathrm{CDCl}_{3}$ ): $\delta 11.59$ (2H, s, OH), $11.29(1 \mathrm{H}, \mathrm{s}, \mathrm{OH}), 7.92$ - $6.76(12 \mathrm{H}$, $\left.\mathrm{m}, \mathrm{H}_{\mathrm{Ar}}\right), 6.02(1 \mathrm{H}, \mathrm{s}, \mathrm{CH})$; MS (m/z): 429 (1), 428 $\left(\mathrm{M}^{+}, 10\right), 307(9), 265$ (42), $162(56), 120$ (100), 92 (61), 76 (18); Anal. Data calc. for $\mathrm{C}_{25} \mathrm{H}_{16} \mathrm{O}_{7}$ : C, 70.09; H, 3.76; O, 26.14; found: C, 70.11; $\mathrm{H}$, $3.74 ; \mathrm{O}, 26.13$.

\section{3, 3'-(3, 4-Dimethoxyphenylmethylene)-bis-4- hydroxy-2H-chromen-2-one (3j)}

Yield: $40 \% ; \mathrm{R}_{f}=0.62$ (ethyl acetate), UV (DMF) $\lambda_{\max }: 302 \mathrm{~nm}$; IR (KBr) $\mathrm{v}_{\max }, \mathrm{cm}^{-1}: 3492,2919$, 1676, 1560; ${ }^{1} \mathrm{HNMR}\left(400 \mathrm{MHz}, \mathrm{CDCl}_{3}\right): \delta 11.64$ $(2 \mathrm{H}, \mathrm{s}, \mathrm{OH}), 7.95-6.90\left(11 \mathrm{H}, \mathrm{m}, \mathrm{H}_{\mathrm{Ar}}\right), 6.07(1 \mathrm{H}$, $\mathrm{s}, \mathrm{CH}), 3.59\left(6 \mathrm{H}, \mathrm{s}, \mathrm{OCH}_{3}\right) ; \mathrm{MS}(\mathrm{m} / \mathrm{z}): 473(2)$, 472 (20), 161 (10) 150 (100), 117 (29), 76 (2); Anal. Data calc. for $\mathrm{C}_{27} \mathrm{H}_{20} \mathrm{O}_{8}: \mathrm{C}, 68.64 ; \mathrm{H}, 4.27$; O, 27.09; found: C, 68.62; H, 4.29; O, 27.09. 


\section{DPPH radical scavenging activity}

The synthesized compounds were evaluated for DPPH (2,2-diphenyl-1-picrylhydrazyl) free radical scavenging activity expressed in term of $I_{50}$ values in Table 2.

\section{Reducing power}

Reducing power of synthesized compounds as a function of concentration is shown in Table 3 . The reducing power assay results showed significant difference $(p<0.01$ and $p<0.001)$ levels among different compounds.

\section{Antioxidant activity in linoleic acid system}

The results for inhibition of linoleic acid peroxidase of synthesized compounds are summarized in Table 4.

\section{DISCUSSION}

A total of ten 3-substituted-bis-2H-chromen-2tones (3a-j) were synthesized as potential antioxidants. The chemical structures of these compounds were confirmed by FT-IR, ${ }^{1} \mathrm{H}$ NMR, mass spectrometry and elemental analysis. The presence of aliphatic - $\mathrm{CH}$ moiety in the title compounds was supported by the appearance of sharp singlet at $\delta(\mathrm{ppm})$ values from 6.01 to 6.10 in the ${ }^{1} \mathrm{H}$-NMR spectra. ${ }^{1} \mathrm{H}$-NMR spectra also confirmed the required number of $\mathrm{H}$-atoms of the title compounds. The presence of IR absorption peaks from $1620-1678 \mathrm{~cm}^{-1}$ were observed due to the presence of $\mathrm{C}=\mathrm{O}$ group in the synthesized compounds. Furthermore, the elemental analysis and molecular ion peaks of the title compounds were found to be consistent with the assigned structures.

The role of antioxidants is to remove free radicals in food and play an important role as a healthprotecting factor.

Table 2: DPPH scavenging activity of biscoumarins based in term of $\mathrm{IC}_{50}$ values

\begin{tabular}{|c|c|c|c|}
\hline Compound no. & $\mathbf{R}^{1}$ & $\mathbf{R}^{2}$ & $\mathbf{I C}_{50}(\mu \mathrm{g} / \mathrm{mL}) \pm \mathrm{S} . \mathrm{D}^{\mathrm{a}}$ \\
\hline $\begin{array}{l}3 a^{*} \\
3 b^{*}\end{array}$ & $\begin{array}{l}-\mathrm{H} \\
-\mathrm{Cl}\end{array}$ & $\begin{array}{l}-\mathrm{H} \\
-\mathrm{H}\end{array}$ & $\begin{array}{l}304.92 \pm 2.32 \\
462.91 \pm 1.57\end{array}$ \\
\hline $3 c^{*}$ & $-H$ & $-\mathrm{Cl}$ & $241.02 \pm 2.47$ \\
\hline $3 d^{*}$ & $-\mathrm{OH}$ & $-\mathrm{OCH}_{3}$ & $159.36 \pm 4.81$ \\
\hline $3 e^{*}$ & $-\mathrm{N}\left(\mathrm{CH}_{3}\right)_{2}$ & $-\mathrm{H}$ & $92.92 \pm 3.23$ \\
\hline $3 f^{*}$ & $-\mathrm{H}$ & $-\mathrm{OH}$ & $116.65 \pm 5.36$ \\
\hline $3 g^{*}$ & $\mathrm{OCH}_{3}$ & $-\mathrm{OH}$ & $53.33 \pm 6.12$ \\
\hline $3 h^{*}$ & $\mathrm{OCH}_{3}$ & $-H$ & $68.44 \pm 2.67$ \\
\hline $3 i^{*}$ & $-\mathrm{OH}$ & $-\mathrm{H}$ & $118.22 \pm 1.23$ \\
\hline $3 \mathbf{j}^{\star}$ & $-\mathrm{OCH}_{3}$ & $-\mathrm{OCH}_{3}$ & $130.94 \pm 5.21$ \\
\hline $\mathrm{BHT}^{*}$ & & & $71.00 \pm 0.82$ \\
\hline
\end{tabular}

${ }^{a}$ Required concentration of the tested compound to scavenge $50 \%$ of DPPH radical; ${ }^{2}$ concentration of $0.1 \mathrm{mM}$; average from 3 assays; * differences were considered significant at $p<0.05$

Table 3: Reducing power of biscoumarins (mean \pm SD)

\begin{tabular}{|c|c|c|c|c|c|}
\hline \multirow{2}{*}{ Compound } & \multirow[b]{2}{*}{$2 \mathrm{mg} / \mathrm{mL}$} & \multicolumn{4}{|c|}{ Reducing power } \\
\hline & & $4 \mathrm{mg} / \mathrm{mL}$ & $6 \mathrm{mg} / \mathrm{mL}$ & $8 \mathrm{mg} / \mathrm{mL}$ & $10 \mathrm{mg} / \mathrm{mL}$ \\
\hline $3 a^{*}$ & $0.08 \pm 0.00$ & $0.09 \pm 0.02$ & $0.11 \pm 0.00$ & $0.12 \pm 0.09$ & $0.13 \pm 0.07$ \\
\hline $3 b^{*}$ & $0.04 \pm 0.15$ & $0.07 \pm 0.03$ & $0.09 \pm 0.03$ & $0.12 \pm 0.07$ & $0.16 \pm 0.13$ \\
\hline $3 c^{*}$ & $0.09 \pm 0.34$ & $0.12 \pm 0.25$ & $0.18 \pm 0.33$ & $0.2 \pm 0.12$ & $0.23 \pm 0.36$ \\
\hline $\begin{array}{l}3 d^{*} \\
3 e^{*}\end{array}$ & $\begin{array}{l}0.11 \pm 0.01 \\
0.12 \pm 0.02\end{array}$ & $\begin{array}{l}0.25 \pm 0.00 \\
0.18 \pm 0.00\end{array}$ & $\begin{array}{l}0.29 \pm 0.60 \\
0.23 \pm 0.14\end{array}$ & $\begin{array}{l}0.38 \pm 0.09 \\
0.26 \pm 0.12\end{array}$ & $\begin{array}{c}0.42 \pm 0.02 \\
0.3 \pm 0.50\end{array}$ \\
\hline $3 f^{\star \star}$ & $0.27 \pm 0.02$ & $0.28 \pm 0.00$ & $0.32 \pm 0.01$ & $0.33 \pm 0.01$ & $0.40 \pm 0.02$ \\
\hline $3 g^{* \star \star}$ & $0.27 \pm 0.02$ & $0.29 \pm 0.01$ & $0.34 \pm 0.01$ & $0.46 \pm 0.01$ & $0.49 \pm 0.01$ \\
\hline $3 h^{\star *}$ & $0.26 \pm 0.00$ & $0.29 \pm 0.01$ & $0.31 \pm 0.07$ & $0.34 \pm 0.00$ & $0.35 \pm 0.01$ \\
\hline $3 i^{\star}$ & $0.2 \pm 0.01$ & $0.2 \pm 0.01$ & $0.22 \pm 0.01$ & $0.24 \pm 0.01$ & $0.26 \pm 0.02$ \\
\hline $3 j^{*}$ & $0.12 \pm 0.03$ & $0.14 \pm 0.00$ & $0.17 \pm 0.02$ & $0.19 \pm 0.01$ & $0.21 \pm 0.00$ \\
\hline$B H T$ & $0.04 \pm 0.00$ & $0.09 \pm 0.00$ & $0.13 \pm 0.00$ & $0.15 \pm 0.00$ & $0.19 \pm 0.00$ \\
\hline
\end{tabular}

The differences were considered significant at $p<0.001\left(^{* * *}\right), p<0.01\left(^{* *}\right)$ and $p<0.05\left(^{*}\right)$ levels 
Table 4: Linoleic acid lipid peroxidation results (mean \pm SD)

\begin{tabular}{|c|c|c|c|c|}
\hline \multirow{2}{*}{ Compound } & \multicolumn{3}{|c|}{ Lipid peroxidation } & \multirow{2}{*}{$\begin{array}{c}\text { Inhibition of linoleic acid } \\
\text { peroxidase (\%) }\end{array}$} \\
\hline & $24 h$ & $48 h$ & $72 h$ & \\
\hline $3 a$ & $0.46 \pm 0.01^{\mathrm{c}}$ & $0.88 \pm 0.00^{c}$ & $1.18 \pm 0.01^{a}$ & 44.2 \\
\hline $3 b$ & $0.54 \pm 0.03^{\mathrm{a}}$ & $1.13 \pm 0.01^{\mathrm{c}}$ & $1.56 \pm 0.01^{b}$ & 41.01 \\
\hline $3 c$ & $0.27 \pm 0.03^{c}$ & $0.49 \pm 0.00^{\mathrm{a}}$ & $1.58 \pm 0.00^{b}$ & 45.32 \\
\hline $3 d$ & $0.3 \pm 0.01^{a}$ & $0.64 \pm 0.01^{b}$ & $0.89 \pm 0.01^{a}$ & 69.87 \\
\hline $3 e$ & $0.34 \pm 0.15^{c}$ & $0.53 \pm 0.07^{c}$ & $0.97 \pm 0.00^{\mathrm{a}}$ & 61.33 \\
\hline $3 f$ & $0.45 \pm 0.03^{c}$ & $0.81 \pm 0.45^{\mathrm{c}}$ & $1.13 \pm 0.05^{c}$ & 63.11 \\
\hline $3 g$ & $0.46 \pm 0.12^{c}$ & $0.77 \pm 0.04^{b}$ & $0.88 \pm 0.12^{c}$ & 70.34 \\
\hline $3 h$ & $0.32 \pm 0.00^{c}$ & $0.66 \pm 0.12^{c}$ & $0.82 \pm 0.00^{c}$ & 63.96 \\
\hline $3 \mathbf{i}$ & $0.44 \pm 0.04^{b}$ & $0.8 \pm 0.32^{c}$ & $0.99 \pm 0.02^{\mathrm{a}}$ & 46.12 \\
\hline $3 \mathbf{j}$ & $0.54 \pm 0.13^{c}$ & $0.67 \pm 0.03^{b}$ & $0.78 \pm 0.02^{c}$ & 39.38 \\
\hline BHT & $0.38 \pm 0.01^{a}$ & $0.62 \pm 0.01^{\mathrm{c}}$ & $1.01 \pm 0.70^{c}$ & 72.27 \\
\hline $\begin{array}{l}\text { Negative } \\
\text { control }\end{array}$ & $0.89 \pm 0.13$ & $1.09 \pm 0.35$ & $1.73 \pm 0.12$ & - \\
\hline
\end{tabular}

There are many reports that suggest the use of antioxidant supplementation in reducing the level of oxidative stress related diseases. This could be achieved by donating the hydrogen to free radical in its reduction to non-reactive species [18]. The addition of hydrogen would remove the odd electron feature which is accountable for free radical reactivity.

The DPPH free radical scavenging activity results of synthesized compounds are given in Table 2. DPPH is a stable organic free radical gives purple color in solution with maximum absorption at $517 \mathrm{~nm}\left(\lambda_{\max }\right)$. Through the DPPH test, compounds have down to reduce the stable DPPH radical to yellow colored diphenylpicrylhydrazine [18]. An interesting trend was observed in the case of compound $\mathbf{3 g}$ having 4-methoxy-3-hydroxyphenyl unit showed highest DPPH scavenging activity. Whereas compound $3 \mathrm{~h}$ having 4-methoxyphenyl moiety was second potent antioxidant compound. After analyzing these trends, the DDPPH inhibition activity could be linked with structure of biscoumarin analogues. It is deduced that electron donating (+ve mesomeric effect) methoxy/hydroxy group at para-position directed the maximum DPPH scavenging activity as in the case of compounds $\mathbf{3 g}$ and $\mathbf{3 h}$ even better than the standard BHT [8]. All the synthesized compounds indicated moderate to excellent DPPH free radical scavenging activity. A proposed mechanism for the DPPH free radical scavenging activity for the compound $3 \mathrm{~g}$ is given in Figure 2 and Figure 3.

The reducing power assay was based on gradual color change by reduction of oxidants as function of reducing agent concentration. Initially, the yellow color of test solution was appeared due to $\mathrm{Fe}^{3+} /$ ferricyanide complex concentration which gradually changes to different shades of green/blue colors on reduction of $\mathrm{Fe}^{3+} / \mathrm{Fe}^{2+}$ as the concentration of antioxidant increases. Herein, reduced concentration of $\mathrm{Fe}^{3+} / \mathrm{Fe}^{2+}$ was determined by measuring absorption of Perl's Prussian blue at $700 \mathrm{~nm}$ [19]. The absorption is directly related to the reducing power and consequently the antioxidant potential.

Compounds $\mathbf{3 g}, \mathbf{3 d}, \mathbf{3 f}, \mathbf{3 h}, \mathbf{3 i}, \mathbf{3 j}, \mathbf{3 e}$ and $\mathbf{3 c}$ showed higher reducing power as compared to standard compound (Table 3). Maximum reducing power (highest absorbance) was exhibited by the compound $\mathbf{3 g}$ with 4-methoxy-3hydroxyphenyl unit due to the electron donating resonance effect of methoxy at the para-position. Statistical analysis showed a significance difference at $p<0.001, p<0.01$ and $p<0.05$ levels.

Lipid peroxidation is also a major cause of food deterioration, affecting colour, flavour, texture and nutritional value [20]. The antioxidant potential of different biscoumarins was evaluated by employing linoleic acid peroxidation system. However, synthesized compounds showed linoleic acid lipid peroxidation inhibition less than the standard BHT (Table 4). All the compounds showed, linoleic acid hydroperoxides inhibition ability in the following decreasing order: $3 g>3 d>3 f>3 h>3 e>3 i>3 c>3 a>3 b>3 j$. The compounds $\mathbf{3 g}$ and $\mathbf{3 d}$ were found to be most active compound to inhibit the linoleic acid peroxides. 
<smiles>O=[N+]([O-])c1cc(N(c2ccccc2)c2ccccc2)c([N+](=O)[O-])c([N+](=O)[O-])c1</smiles>

Purple color

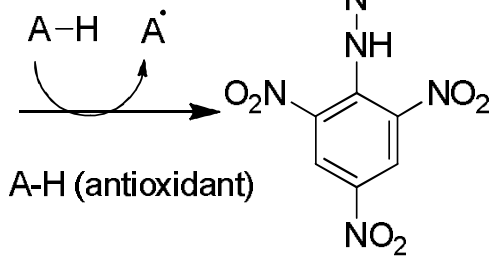

DPPH-H<smiles>COc1ccc(C(c2c(O)c3ccccc3oc2=O)c2c(O)c3ccccc3oc2=O)cc1OP</smiles><smiles>[TeH][Te]P[PbH]</smiles>

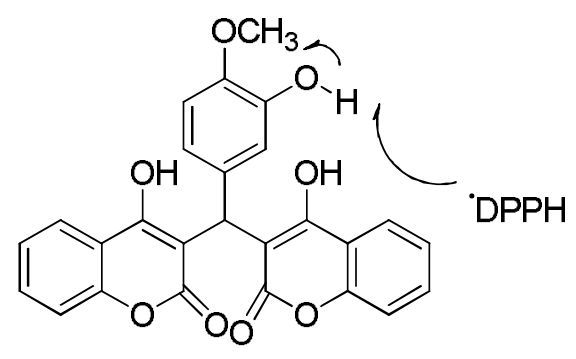

Compound $\mathbf{3 g}$ (antioxidant)<smiles></smiles>

Radical stablized through resonance

Figure 2: Proposed mechanism for DPPH free radical scavenging activity by compound $\mathbf{3 g}$

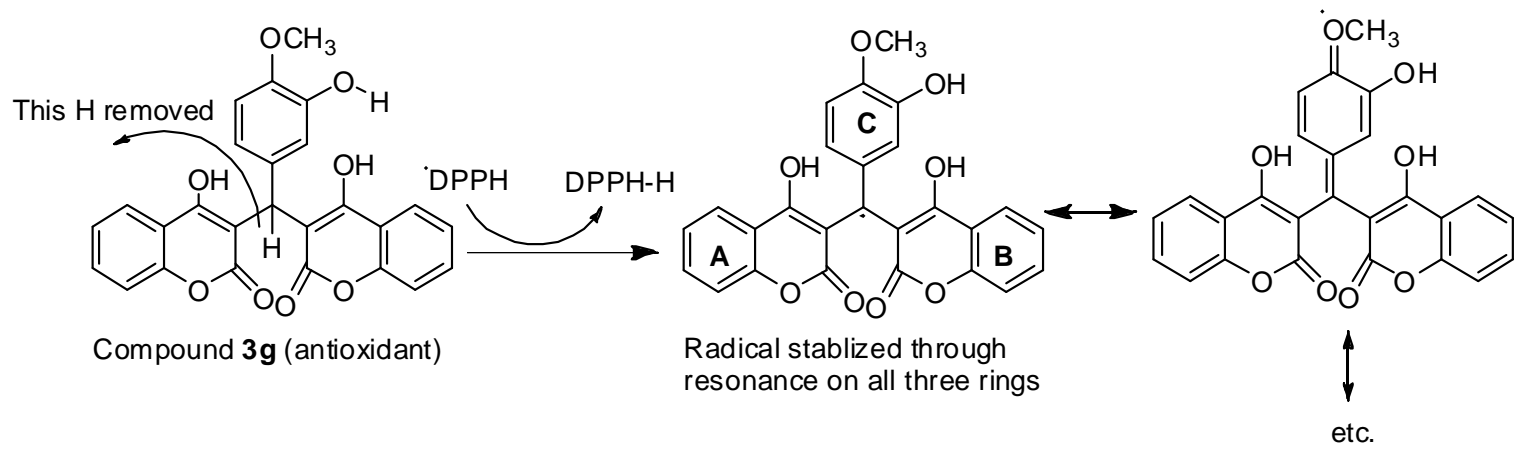

Figure 3: Second proposed mechanism for DPPH free radical scavenging activity of compound $\mathbf{3 g}$

\section{CONCLUSION}

The search for new compounds with antioxidant properties has been a very dynamic area of research. Compound $3 \mathrm{~g}$ showed good antioxidant activity in all the methods used during this study. Among the tested compounds 3,3'(3-hydroxy-4-methoxybenzylidene)-bis-(4hydroxycoumarin) (3g) exhibited the highest antioxidant activity. The results suggest that a correlation exists between antioxidant activity with the position, number and electronic nature of substituents present on the aryl moiety of biscoumarins. Further, the presence of electron donating substituents such as methoxy/hydroxy at para-position and the meta-position of aryl moiety in the case of compounds $\mathbf{3} \mathbf{g}$ and $\mathbf{3 d}$, is essential for enhanced antioxidant activity.

\section{DECLARATIONS}

\section{Acknowledgement}

The authors are grateful to Higher Education Commission of Pakistan (HEC) for providing financial support and laboratory facilities for the work.

\section{Conflict of Interest}

No conflict of interest associated with this work. 


\section{Contribution of Authors}

The authors declare that this work was done by the authors named in this article and all liabilities pertaining to claims relating to the content of this article will be borne by them.

\section{Open Access}

This is an Open Access article that uses a funding model which does not charge readers or their institutions for access and distributed under the terms of the Creative Commons Attribution License (http://creativecommons.org/licenses/by 14.0) and the Budapest Open Access Initiative (http://www.budapestopenaccessinitiative.org/rea d), which permit unrestricted use, distribution, and reproduction in any medium, provided the original work is properly credited.

\section{REFERENCES}

1. Finkel $T$, Holbrook NJ. Oxidants, oxidative stress and the biology of ageing. Nature 2000; 408: 239-247.

2. Mandal S, Yadav S, Yadav S, Nema RK. Antioxidants: $A$ review. J Chem Pharm Res 2009;1(1):102-104.

3. Hussain Al, Shahzad ASC, Sumera N, Muhammad UA, Zulfiqar AK, Hassaan AR, Munavvar ZAS. Effect of Extraction Techniques and Solvent Systems on the Extraction of Antioxidant Components from Peanut (Arachis hypogaea L.) Hulls. Food Anal Meth 2012; 5: 890-896.

4. Alam MN, Bristi NJ, Rafiquzzaman M. Review on in vivo and in vitro methods evaluation of antioxidant activity. Saudi Pharm J 2013; 21: 143-152.

5. Musa MA, Cooperwood JS, Khan MO. A review of coumarin derivatives in pharmacotherapy of breast cancer. Curr Med Chem 2008; 15(26): 2664-2679.

6. Perron NR, Julia L. Brumaghim Review of the antioxidant mechanisms of polyphenol compounds related to iron binding. Cell Biochem Biophys 2009; 53: 75-100.

7. Hussain $H$, Javed $H$, Ahmad $A H$, Karsten $K$. The chemistry and biology of bicoumarins. Tetrahedron 2012; 68: 2553-2578.

8. Khan KM, lqbal S, Lodhi MA, Maharvi GM, Ullah Z, Choudhary MI, Rahman AU, Perveen S. Biscoumarin: new class of urease inhibitors; economical synthesis and activity. Bioorg \& Med Chem 2004; 12(8): 19631968.

9. Choudhary MI, Fatima N, Khan KM, Jalil S, lqbal S, Rehman AU. New biscoumarin derivatives-cytotoxicity and enzyme inhibitory activities. Bioorg \& Med Chem 2006; 14(23): 8066-8072.

10. Kancheva VD, Boranova PV, Nechev JT, Manolov II. Structure-activity relationships of new 4-hydroxy biscoumarins as radical scavengers and chain-breaking antioxidants. Biochimie 2010; 92(9): 1138-1146.

11. Lacy A, Richard OK. Studies on Coumarins and Coumarin-Related Compounds to Determine their Therapeutic Role in the Treatment of Cancer. Curr Pharm Design 2004; 10: 3797-3811.

12. Chiang CC, Mouscadet JF, Tsai HJ, Liu CT, Hsu LY. Synthesis and HIV-1 integrase inhibition of novel bis- or tetra-coumarin analogues. Chemical Pharm Bull 2007; 55(12): 1740-1743.

13. Arora KR, Navneet K, Yogita B, Gulshan B. Novel coumarin benzimidazole derivativesas antioxidants and safer anti-inflammatory agents. Acta Pharm Sinica $B$ 2014; 4(5): 368-375.

14. Kancheva VD, Luciano S, Petya VB, Abdullah K, Manju $K S$, Mukesh KP, Shashwat M, Jordan ZN, Sunil KS, Ashok KP, et al. Structure-activity relationship of dihydroxy-4-methylcoumarins as powerful antioxidants: Correlation between experimental \& theoretical data and synergistic effect. Biochimie 2010; 92: 1089-1100.

15. Osman H, Afsheen, A, Chan KL, Mark CB. Microwaveassisted synthesis and antioxidant properties of hydrazinyl thiazolyl coumarin derivatives. Chem Cent $J$ 2012; 6(1): 32-41.

16. Isabel CFRF, Paula B, Miguel VB, Lillian B. Free-radical scavenging capacity and reducing power of wild edible mushrooms from northeast Portugal: Individual cap and stipe activity. Food Chem 2007; 100: 1511-1516.

17. Halliwell B, John G. Free radicals in biology and medicine, ed 3, Oxford: Oxford University Press, 1999.

18. Amarowicz R, Pegg RB, Rahimi PM, Barl B, Weil JA. Free-radical scavenging capacity and antioxidant activity of selected plant species from the Canadian prairies. Food Chem 2004; 5: 551-562.

19. Rahmat AK. Evaluation of flavonoids and diverse antioxidant activities of Sonchusarvensis. Chem Cent $J$ 2012; 6: 126-132.

20. Yen GC, Hui-Yin C. Antioxidant activity of various tea extracts in relation to their antimutagenicity. J Agri \& Food Chem 1995; 43: 27-32. 